\title{
Evaluación del estado nutricio del adulto mayor asilado en la ciudad de Aguascalientes, México
}

\author{
Evaluation of the nutritional status of the older adults living in nursing \\ homes in the city of Aguascalientes, Mexico
}

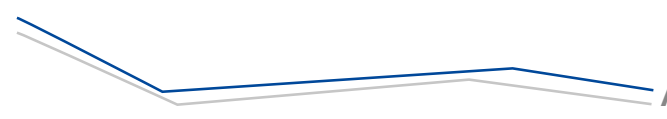

Abril Alejandra Rivera-Flores*, Valeria Díaz-Posada*, Jessica Díaz-López*, Judith Martín del Campo-Cervantes**⿶

Rivera-Flores, A. A., Díaz-Posada, V., Díaz-López, J., \& Martín del Campo-Cervantes, J. (2020). Evaluación del estado nutricio del adulto mayor asilado en la ciudad de Aguascalientes, México. Investigación y Ciencia de la Universidad Autónoma de Aguascalientes, 28(81), 66-73.

\section{RESUMEN}

El adulto mayor (AM) institucionalizado está en riesgo de padecer desnutrición, la cual aumenta el riesgo de desarrollar comorbilidades que la convierten en un problema de salud pública. En el presente trabajo se evaluó el estado nutricio del AM mediante la Evaluación mini nutricional (por sus siglas en inglés MNA), un recordatorio dietético de $24 \mathrm{~h}$ y la toma de medidas antropométricas. Los resultados mostraron que $56.7 \%$ de la población estudiada presentó riesgo de malnutrición de acuerdo al MNA. Se encontró dependencia funcional en $86.6 \%$ de los participantes al presentar una fuerza muscular baja, además de una glucemia capilar alterada con un promedio de $145.66 \mathrm{mg} / \mathrm{dl}$ y un índice de masa corporal promedio de $24.62 \mathrm{~kg} / \mathrm{m}^{2}$. La dieta del AM fue deficiente en energía, proteínas y fibra, lo que podría explicar la pérdida de masa magra y funcionalidad.

Palabras clave: adulto mayor; estado nutricio; MNA; asilo.

Keywords: older adult; nutritional status; MNA; nursing home.

Recibido: 18 de mayo de 2020, aceptado: 22 de octubre de 2020

* Licenciatura en Nutrición, Universidad Autónoma de Aguascalientes. Av. Universidad \# 940, Ciudad Universitaria, C. P. 20131, Aguascalientes, Ags., México. Correo electrónico: abrilfoores51@gmail.com; valeria. posada44@gmail.com; jesua.diaz.5L@gmail.com ORCID: http://orcid. org/0000-0001-6640-0523; http://orcid.org/0000-0003-0176-3942; http://orcid.org/0000-0002-0561-7371

** Departamento de Nutrición, Centro de Ciencias de la Salud, Universidad Autónoma de Aguascalientes. Av. Universidad \# 940, Ciudad Universitaria, Edificio 107 planta baja, C. P. 20131, Aguascalientes, Ags., México. Correo electrónico: jmartic@correo.vaa.mx DOI: http://orcid. org/0000-0003-1855-6309

$\square$

Autora para correspondencia
ABSTRACT

The institutionalized elderly's are at risk of malnutrition, which increases the risk of developing comorbidities making it a public health problem. In this paper, we evaluated the nutritional status of the older adults with the Mini Nutritional Assessment (MNA), a 24-h dietary recall and anthropometric measurements. $56.7 \%$ of the participants showed nutritional risk according to the MNA. Dependence was found in $86.6 \%$ of the participants by presenting a low muscular strength, in addition to an altered capillary glycemia with an average of $145.66 \mathrm{mg} / \mathrm{dl}$ and an average body mass index of $24.62 \mathrm{~kg} / \mathrm{m}^{2}$. The elderly's diet was energy, protein and fiber deficient; which consequently can cause loss of lean mass and functionality.

INTRODUCCIÓN

De acuerdo con las estimaciones del Consejo Nacional de Población (CONAPO) realizadas con base en los resultados de la encuesta intercensal de 2015 del Instituto Nacional de Estadística y Geografía (INEGI), el estado de Aguascalientes tenía una población de 119,691 habitantes de 60 años o más. Para el segundo semestre de 2017 los adultos mayores de 60 años representaban $9 \%$ de la población; las proyecciones poblacionales contemplan que para 2030 el porcentaje aumentará a 13.5\%, sumando más de 200,000 adultos mayores (Alarcón Sánchez, Pinto López, \& Benalcázar Game, 2018).

El adulto mayor (AM) es un grupo etario en riesgo de padecer desnutrición a causa de factores sociales y psicológicos, alteraciones metabólicas, enfermedades crónico-degenerativas, polifarmacia 
y deterioro de la capacidad funcional. La desnutrición desencadena múltiples efectos en el cuerpo, como la disminución del funcionamiento del tracto gastrointestinal, deterioro del sistema inmune, entre otros efectos que aumentan el riesgo de presentar o descontrolar comorbilidades, lo que la convierte en un problema de salud pública (Alvarado-García, Lamprea-Reyes, \& Murcia-Tabares, 2017).

En el área geriátrica el manejo nutricional debe individualizarse tomando en cuenta aspectos propios del envejecimiento, con el objetivo de proporcionar a través de la dieta los nutrimentos necesarios en las cantidades adecuadas para mantener un buen estado nutricio; por ello es necesaria la participación de los profesionales para evaluar el estado de nutrición del adulto mayor, apoyándose con herramientas de tamizaje específicas e indicadores nutricionales y elaborar el plan de intervención en beneficio de la salud del adulto mayor (Burgos Vélez, González Solís, \& Castro Posligua, 2018).

El objetivo de esta investigación fue evaluar el estado de nutrición de adultos mayores asilados en el estado de Aguascalientes.

MATERIALES Y MÉTODOS

Estudio descriptivo, observacional y analítico donde se evaluó a adultos mayores de 60 años de ambos géneros residentes de asilos en la ciudad de Aguascalientes; por la naturaleza observacional del estudio no se generó hipótesis de investigación. Se excluyeron AM con marcapasos, demencia senil o Alzheimer, o que no permitieron la toma capilar de glucosa ni de tensión arterial, así como aquellos a quienes se les dificultó permanecer de pie para la toma de medidas antropométricas. La muestra se obtuvo de forma no probabilística por cuota. Se utilizó el sistema software NutriKcalßVO para ingresar los datos del recordatorio de $24 \mathrm{~h}$ y obtener la cantidad consumida de energía, proteína, lípidos, hidratos de carbono y micronutrimentos, como calcio y fibra.

Se aplicó la Evaluación mini nutricional (MNA, por sus siglas en inglés), un recordatorio de $24 \mathrm{~h}$ del consumo de alimentos, un cuestionario para la obtención de datos personales; se tomaron medidas antropométricas (peso, talla, circunferencia media de brazo, circunferencia de pantorrilla y fuerza de prensión de mano), la glucosa capilar y la tensión arterial.

Para evaluar el estado nutricio se hizo uso del MNA, herramienta de tamizaje para identificar malnutrición en el adulto mayor con tres clasificaciones: normal, riesgo de desnutrición y desnutrición; para valorar la fuerza muscular se utilizó la prueba de fuerza de prensión de mano a través de un dinamómetro mecánico marca TAKEI Smedley III T-18A precisión 1 kg, y la masa muscular se determinó a través de la bioimpedancia eléctrica con una báscula de bioimpedancia marca TANITA BC-558 Ironman Segmental; para la determinación de glucosa capilar se utilizó un glucómetro marca SDcheck Gold y para la medición de la tensión arterial un baumanómetro automático de brazalete digital Omron HEM-7121. Para la medición de estatura se utilizó un estadímetro marca SECA 213 precisión $1 \mathrm{~mm}$ y para la toma de la circunferencia media de brazo y pantorrilla una cinta métrica de fibra de vidrio marca Lufkin precisión $1 \mathrm{~mm}$.

El análisis estadístico se realizó con el software IBM SPSS Statistics versión 25.0, para realizar la estadística descriptiva (media, desviación estándar) e inferencial (prueba t no pareada y prueba U MannWhitney); considerando un nivel de significancia con valor de $p<0.05$.

\section{RESULTADOS}

\section{Características demográficas}

Se evaluaron 30 adultos mayores, $73 \%$ fueron mujeres $(n=22)$ y $27 \%$ fueron hombres $(n=8)$, residentes de cinco asilos privados de la ciudad de Aguascalientes: Asilo José Guadalupe Alcalá, A. C. (siete adultos), Casa hogar San José (cuatro adultos), El hogar del anciano (10 adultos), Estancia de vida de nuestra señora de Guadalupe, A. C. (cinco adultos) y Casa hogar Cuicacalli (cuatro adultos); con una edad promedio de 80 años (hombres: $78.75 \pm 6.84$ años de edad, mujeres: $81 \pm 6.88$ años de edad) con edades fluctuando entre los 67 a 94 años de edad.

\section{Estado nutricio y características antropométricas}

En porcentaje, $36.7 \%$ de la población presentó estado nutricio normal, mientras que $63.3 \%$ presentó riesgo o desnutrición de acuerdo con el MNA (tabla 
1). El índice de masa corporal tuvo una media de $24.62 \mathrm{~kg} / \mathrm{m}^{2}$ (DE $\pm 4.60 \mathrm{~kg} / \mathrm{m}^{2}$ ); al clasificarlo usando los puntos de corte para adulto mayor se encontró a 11 adultos mayores (36.7\%) con estado normal de nutrición, siete con sobrepeso (23.3\%), dos con obesidad (6.7\%) y 10 con desnutrición (33.3\%).
El promedio poblacional de peso corporal fue $57.57 \mathrm{~kg}(D E \pm 12.903 \mathrm{~kg})$ y de talla fue $1.52 \mathrm{~m}$ (DE $\pm 0.09 \mathrm{~m}$ ); se encontró que los hombres tuvieron un mayor peso corporal $(p=0.023)$ y talla $(p=0.000)$ en comparación con las mujeres. La fuerza muscular fue mayor en hombres que en mujeres ( $p=0.008$ ); los demás parámetros no presentaron una diferencia entre géneros estadísticamente significativa (tabla 2).

Tabla 1

Clasificación de la evaluación mini nutricional (MNA)

\begin{tabular}{lll}
\hline PUNTAJE & CLASIFICACIÓN & $\begin{array}{l}\text { FRECUENCIA } \\
\text { (PORCENTAJE) }\end{array}$ \\
\hline $24-30$ & Estado de nutrición normal & $11(36.7 \%)$ \\
$17-23.5$ & Riesgo de desnutrición & $17(56.7 \%)$ \\
$\leq 16.5$ & Desnutrición & $2(6.6 \%)$ \\
\hline $\begin{array}{l}\text { Nota: } n=30 \text { adultos mayores. } \\
\text { Elaboración propia. }\end{array}$ & & \\
\hline
\end{tabular}

Tabla 2

Características antropométricas poblacionales y por género

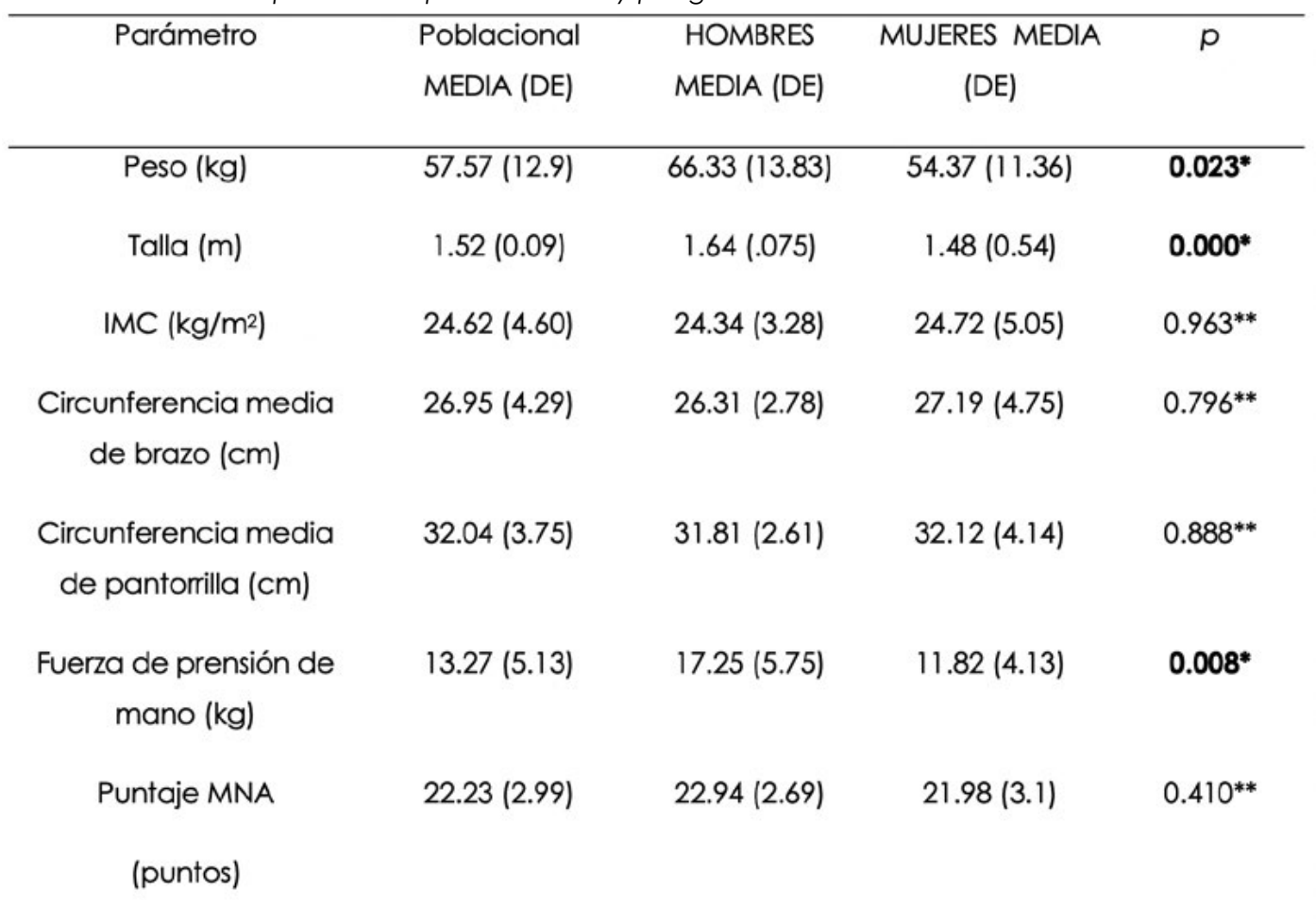

Nota: $n=30$ adultos mayores (22 mujeres, 8 hombres). DE: Desviación estándar. Valores de $p$ usando *Prueba $†$ no pareada $\bigcirc * *$ Prueba $\cup$ de Mann-Whitney para comparar diferencias entre hombres y mujeres.

Elaboración propia. 


\section{Composición corporal}

Al comparar la masa muscular se encontró que los hombres presentaron $10.64 \mathrm{~kg}$ más de músculo que las mujeres $(p=0.000)$. Por otro lado, las mujeres presentaron $13.1 \%$ más de porcentaje de grasa corporal que los hombres (tabla 3). La glucosa capilar tuvo un promedio de $145.66 \mathrm{mg} / \mathrm{dl}(D E \pm 60.52 \mathrm{mg} / \mathrm{dl}$ ).

\section{Dependencia}

La mns del adulto mayor se valoró mediante la fuerza de prensión de mano, la cual fue estadísticamente mayor ( $t=-2.864, p=0.008)$ para los hombres que para las mujeres (tabla 2), al presentar $5.43 \mathrm{~kg}$ más de fuerza muscular. La dependencia funcional evaluada por este parámetro se encontró en $86.7 \%(n=26)$ de los participantes, lo que indica que $13.3 \%(n=4)$ fueron independientes.

Tabla 3

Composición corporal poblacional y por género

\begin{tabular}{ccccc}
\hline Parámetro & $\begin{array}{c}\text { Poblacional } \\
\text { MEDIA (DE) }\end{array}$ & $\begin{array}{c}\text { HOMBRES } \\
\text { MEDIA (DE) }\end{array}$ & $\begin{array}{c}\text { MUJERES MEDIA } \\
\text { (DE) }\end{array}$ & $p$ \\
\hline Porcentaje de grasa (\%) & $31.62(7.20)$ & $27.81(5.27)$ & $33(7.41)$ & $0.081^{*}$ \\
Masa magra (kg) & $37.17(6.79)$ & $44.97(6.94)$ & $34.33(3.99)$ & $\mathbf{0 . 0 0 0 ^ { * * }}$ \\
Hueso (kg) & $2.01(0.35)$ & $2.42(0.32)$ & $1.85(0.21)$ & $\mathbf{0 . 0 0 0 ^ { * * }}$ \\
Porcentaje de agua (\%) & $46.85(9.36)$ & $45.45(16.58)$ & $47.36(5.33)$ & $0.174^{* *}$ \\
Glucosa capilar (mg/dl) & $145.66(60.52)$ & $146.38(72.01)$ & $145.41(57.69)$ & $0.250^{* *}$
\end{tabular}

Nota: $n=30$ adultos mayores (22 mujeres, 8 hombres). DE: Desviación estándar. Valores de $p$ usando *Prueba $†$ no pareada o **Prueba $U$ de Mann-Whitney para comparar diferencias entre hombres y mujeres.

Elaboración propia.

\section{Consumo dietético}

El promedio del consumo energético ingerido referido por los participantes (figura 1) fue $1017.7 \pm$ 374.05 calorías por día, con una diferencia mínima entre hombres y mujeres (hombres: 949.63 calorías, $D E \pm 363.55$ calorías; mujeres: 1042 calorías, $D E \pm 383$ calorías). El consumo de proteínas fue mayor en las mujeres (41.40 g, DE $\pm 5.05 \mathrm{~g}$ ) que en los hombres (39.93 $\mathrm{g}, D E \pm 11.16 \mathrm{~g})$; mientras que poblacionalmente presentaron una media de $41.01 \mathrm{~g}$ por día (DE \pm 4.39 g por día).
Para el consumo de calcio se obtuvo un promedio poblacional de $364.56 \mathrm{mg}$ por día (DE \pm 168.23 mg por día); este micronutrimento tuvo un consumo parecido en hombres (351.12 mg por día, $D E \pm 196.41$ mg por día) y en mujeres (369.45 mg por día, $D E \pm$ $161.65 \mathrm{mg}$ por día). Para la fibra el promedio de consumo en la población fue de $20.10 \mathrm{~g}$ por día (DE \pm $12.20 \mathrm{~g}$ al día), misma que tuvo una media de consumo en hombres de $21.23 \mathrm{~g}$ al día (DE $\pm 10.84 \mathrm{~g}$ al día) y en mujeres fue de $19.69 \mathrm{~g}$ al día (DE $\pm 12.88 \mathrm{~g}$ al día). No se encontraron diferencias significativas entre hombres y mujeres para ninguno de los valores antes mencionados, lo que implica que el consumo fue igual entre géneros. 


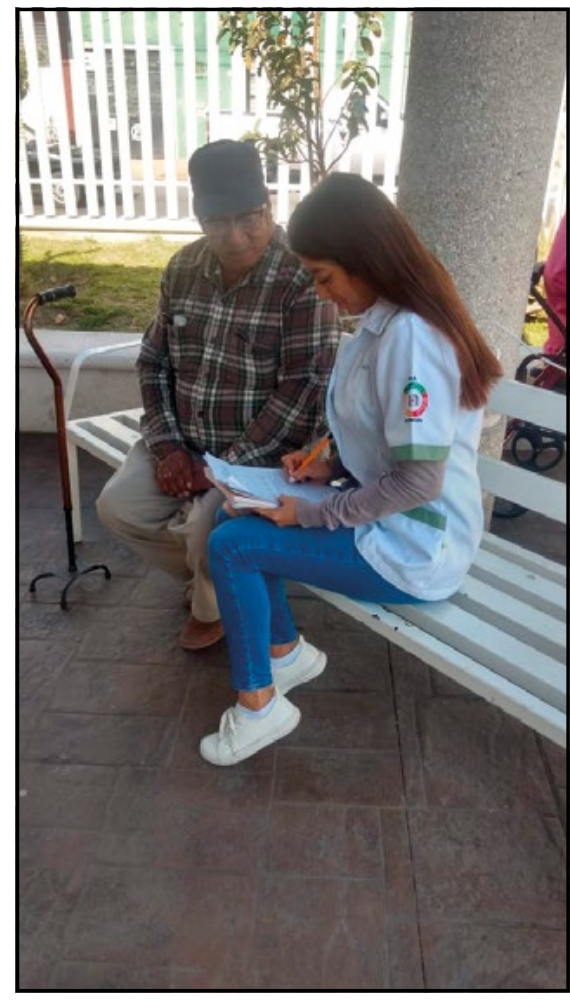

Figura 1. Aplicación de encuestas nutricionales y recordatorio de $24 \mathrm{~h}$.

Fotografía proporcionada por Abril Alejandra Rivera-Flores.

DISCUSIÓN

Al comparar los datos obtenidos por el MNA y el IMC en la población evaluada en relación con la clasificación del estado nutricio se encontró que $6.6 \%$ de la población estudiada presentó desnutrición, cifra que fue mayor con el IMC al encontrarse en $33.33 \%$ de los participantes; lo anterior puede deberse a que el MNA tiene una clasificación de riesgo de desnutrición (encontrada en $56.7 \%$ de la población), en donde se incluye a personas con desnutrición leve y con probabilidad de generarla. Estas diferencias en la clasificación del estado nutricio se deben a la especificidad y sensibilidad del MNA al ser un instrumento creado específicamente para adultos mayores (Calleja Fernández, Vidal Casariego, Cano Rodríguez, \& Ballesteros Pomar, 2016); mientras que el IMC es un indicador poblacional general que no considera la edad de la persona evaluada, los cambios fisiológicos, las comorbilidades, las fracturas, la desmineralización ósea o cualquier otro factor que pudiese sobreestimar el índice de masa corporal y dar como resultado un diagnóstico nutricio erróneo (Calero Saa \& Chaves García, 2016).
Osuna-Padilla, Verdugo-Hernández, Leal-Escobar y Osuna-Ramírez (2015) informaron porcentajes parecidos a los de nuestra población al utilizar el MNA para clasificar el estado nutricio; en su investigación $32.1 \%$ de adultos mayores presentaron desnutrición, $57.1 \%$ riesgo de desnutrición y $10.7 \%$ un estado de nutrición normal. Este estudio presentó mayor cantidad de individuos con desnutrición en comparación con la población evaluada en el presente artículo; este resultado se puede deber a que en el estudio de Osuna-Padilla et al. (2015) los AM eran ambulatorios, lo que implica que la alimentación en esta población es muy diferente a la de las personas asiladas en virtud de que tienen mayor libertad al momento de elegir alimentos, además se tienen que considerar las diferencias sociodemográficas que influyen en el entorno de cada AM debido a que los participantes de Osuna-Padilla et al. (2015) son habitantes de una ciudad del norte de México y Aguascalientes tiene condiciones económicos y demográficas muy diferentes a los estados ubicados en el norte del país; otro aspecto a considerar es que la mayoría de los participantes del estudio de OsunaPadilla et al. (2015) eran hombres, mientras que en la población estudiada en esta investigación predominan las mujeres.

Por otro lado, el estudio de Méndez Estévez et al. (2013) muestra a $44 \%$ de la población con riesgo de desnutrición; de igual manera Interiano Rodas y Valdez (2017) lo identificaron en $56.9 \%$ de sus participantes. Los datos obtenidos por este autor son semejantes a lo encontrado en nuestra población, lo cual puede deberse a que se evaluó a población asilada y no ambulatoria. Lo anterior muestra que la desnutrición es una patología que se encuentra comúnmente en los AM, misma que debe ser identificada y atendida precozmente. La población que participó en el presente estudio mostró una masa muscular deficiente; sin embargo, los hombres presentaron mayor masa magra en comparación con las mujeres, persistiendo la distribución usual de la composición corporal del adulto joven (García Rodríguez, García Vilau, \& Odio Collazo, 2017).

Con relación a la grasa corporal en el adulto mayor, no existe un criterio de corte para el porcentaje adecuado que deben tener hombres y mujeres. Estudios en México han informado un intervalo de entre $37 \%$ y $50 \%$ de grasa corporal en mujeres (González-Pérez, Raya-Giorguli, Botello-Pacheco, \& Rangel-Peniche, 2013). El porcentaje de masa grasa en los AM estudiados fue mayor en $5.19 \%$ para las 
mujeres en comparación con el porcentaje presentado por los hombres, lo que muestra que en edades avanzadas se sigue manteniendo el dimorfismo sexual esperado para cada género.

En el estudio de Peña-Ordóñez, BustamanteMontes, Ramírez-Durán, Halley-Castillo y García-Cáceres (2016) la población de AM fue clasificada en dos grupos con base en pruebas de consumo dietético, presencia de sarcopenia y fuerza; obtuvieron una media poblacional del porcentaje de masa grasa de $30.3 \%$ y $40.2 \%$ para cada grupo. El promedio del porcentaje de masa grasa en el presente estudio fue de $31.6 \%$, resultandos similares a los descritos en el estudio anterior; lo cual puede deberse a que en ambos estudios la cantidad de mujeres fue mayor en comparación con los hombres.

La sarcopenia se presenta en la mayoría de los adultos mayores y tiene consecuencias como disminución de las capacidades físicas, esto favorece una mala calidad de vida y predispone a la muerte (Peña-Ordóñez et al., 2016). La circunferencia de pantorrilla es utilizada para detectar sarcopenia, debido a que presenta una buena relación entre la fuerza muscular y la masa libre de grasa (Cuervo et al., 2009).

Zayas Somoza, Fundora Álvarez y Santana Porbén (2018) aseguran que la circunferencia de pantorrilla es un método de medición antropométrica útil en la detección de sarcopenia. En su investigación el punto de corte utilizado para la circunferencia de pantorrilla fue menor a $31 \mathrm{~cm} ; 46.6 \%$ de la población estudiada presentó menos de $31 \mathrm{~cm}$ en dicho parámetro, lo que puede ayudar a identificar la sarcopenia en los participantes de la presente investigación.

El estudio de Landi, Loperoti y Onder (2013) recomienda estimar la masa muscular a través de indicadores antropométricos como la circunferencia de pantorrilla cuando no se tiene disponible un equipo de composición corporal. En este estudio se identificó una asociación entre la masa muscular y la circunferencia de la pantorrilla, es decir que mientras la masa muscular es menor la circunferencia de pantorrilla también lo es.

El porcentaje de adultos mayores clasificados como dependientes en el presente estudio que necesitaban ayuda para realizar las actividades cotidianas fue de $86.6 \%$. Interiano Rodas y Valdez (2017) clasificaron a $40 \%$ de los AM estudiados como dependientes y a $59.7 \%$ como independientes; como se puede observar en la población estudiada en esta investigación la cantidad de AM dependientes fue mayor, probablemente por el método utilizado debido a que en el estudio de Interiano Rodas y Valdez (2017) utilizaron la escala de Barthel donde se categoriza al AM con independencia, dependencia leve, moderada, severa o total; mientras que en la presente investigación se utilizó la fuerza de prensión de mano obtenida por dinamometría para clasificarlos como dependiente o independiente.

Rodríguez Quintana et al. (2019) clasificó la capacidad funcional con base en las actividades básicas e instrumentales de la vida diaria como trabajar, alimentarse, bañarse, vestirse, etc., de acuerdo con ello identificó a $93.1 \%$ de los AM como dependientes en relación con actividades instrumentales y a $73.5 \%$ como dependientes en actividades básicas. HerazoBeltrán et al. (2017) encontró un grado de dependencia funcional entre moderado y severo para las mujeres y en el género masculino la dependencia fue severa.

Estos resultados son parecidos a los observados en la investigación realizada en los asilos de la ciudad de Aguascalientes, donde se encontró a la mayoría de los adultos mayores con dependencia. Con lo anterior es posible inferir que, a pesar de la diversidad en los métodos para identificar dependencia, esta es una condición que se presenta en la mayoría de los AM, por lo que se deben de generar estrategias para su prevención y tratamiento oportuno.

La fuerza muscular está directamente relacionada con la cantidad de masa muscular, al perder esta en el envejecimiento se reduce la fuerza $y$, por ende, la funcionalidad. Este proceso es progresivo, el músculo presenta cambios anatómicos con el paso del tiempo que pueden ser influenciados por los hábitos alimenticios, factores endocrinos como la disminución en la producción de insulina y el aumento de la resistencia periférica de dicha hormona, además de la disminución en la biodisponibilidad de estrógenos o testosterona (Deossa-Restrepo, Restrepo-Betancur, Velásquez-Vargas, \& Varela-Álvarez, 2016). La fuerza muscular se puede mantener o aumentar con diversos esquemas de ejercicio, mismos que pueden ser específicos para la condición física de cada AM. 
De acuerdo con los hábitos alimenticios referidos por la población estudiada el consumo energético para hombres y mujeres no presentó una diferencia significativa, lo que indica que el género no es un factor para tener una mayor o menor ingesta energética; es importante señalar que el consumo ideal de proteína para AM debería ser de 1 gramo por kg de peso (Calero Saa \& Chaves García, 2016); al ser la media de peso en esta población de 57.57 $\mathrm{kg}$ y el consumo de proteínas de $41.0 \mathrm{~g}$, indica que el aporte es de aproximadamente $0.7 \mathrm{~g}$ de proteína por kg de peso corporal; con este déficit se puede generar una disminución en la masa magra del AM y desencadenar sarcopenia, dependencia y desnutrición.

La población de AM de asilados de la ciudad de Aguascalientes presentó una media de glucosa capilar de $146.66 \mathrm{mg} / \mathrm{dl}$, concentraciones que se mantuvieron parecidos en ambos sexos. Cabe mencionar que únicamente $30 \%$ de esta población tenía un diagnóstico de diabetes previo a la intervención del estudio. Merodio Pérez, Rivas Acuña y Martínez Serrano (2015) evaluaron la glucosa de una población de $A M$, respecto al género se apreció que $64.7 \%$ de los hombres y $60.8 \%$ de las mujeres presentaron descontrol en la concentración de glucosa sanguínea.

En la población estudiada llama la atención que la mayoría de los participantes presentó un descontrol en la concentración de glucosa, son pocos los AM que mantienen concentraciones óptimas de este parámetro bioquímico; el descontrol puede ser consecuencia de una dieta inadecuada, la disminución de la producción de insulina y la resistencia periférica a dicha hormona provocada por el envejecimiento (García Salcedo, Serrano Gallardo, Recio Vega, \& Calderón Salinas, 2011).

Con los resultados obtenidos se hace evidente la necesidad de identificar oportunamente al AM en riesgo nutricio; así como de desarrollar programas nutricionales específicos para AM que permitan cubrir el aporte proteico-energético de la población con la finalidad de disminuir el riesgo o presencia de desnutrición. Asimismo, se deben considerar algunas variables de predicción para la pérdida de peso, como: inapetencia, polifarmacia, falta de piezas dentales, síndromes de malabsorción, sedentarismo, alteraciones en el sentido del olfato y gusto, etc.; mismas que pueden identificarse fácilmente y tratarse de forma oportuna para mejorar el estado nutricio del AM.

CONCLUSIONES

El $63.3 \%$ de la población de AM de la ciudad de Aguascalientes que vive en asilos presentó riesgo de desnutrición o desnutrición y $86.7 \%$ se identificó con dependencia.

El adulto mayor requiere un cuidado específico y un tratamiento integral e individualizado para corregir las deficiencias nutrimentales que pueda presentar; prevenir o tratar la desnutrición, el descontrol de las comorbilidades y la aparición de nuevas enfermedades.

Es necesario generar en el estado de Aguascalientes programas sociales que se enfoquen en la atención multidisciplinaria de este grupo etario y promover la creación de lugares donde se pueda brindar en conjunto la parte sociocultural (clubes del abuelo) y de salud multidisciplinaria (medicina, nutrición, terapia física, psicológica, etcétera) al mismo tiempo.

\section{REFERENCIAS}

- Alarcón Sánchez, M. N., Pinto López, L. P., \& Benalcázar Game, J. (2018). Implementar técnicas nutricionales para adultos mayores desnutridos. Revista Científica Mundo de la Investigación y el Conocimiento, 2(esp.), 435-463. doi: 10.26820/recimundo/2.esp.2018.435-463

- Alvarado-García, A., Lamprea-Reyes, L., \& Murcia-Tabares, K. (2017). La nutrición en el adulto mayor: Una oportunidad para el cuidado de enfermería. Enfermería Universitaria, 14(3), 199-206. doi: 10.1016/j.reu.2017.05.003

- Burgos Vélez, M. J., González Solís, M. M., \& Castro Posligua, A. (2018). Evaluación del estado nutricional de adultos mayores del asilo Carlos Luis Plaza Dañín de Guayaquil (Tesis de pregrado). Recuperada de http://repositorio.ug.edu.ec/ handle/redug//35310

- Calero Saa, P. A., \& Chaves García, M. A. (2016). Cambios fisiológicos de la aptitud física en el envejecimiento. Revista Investigación en Salud Universidad de Boyacá, 3(2), 176-194. doi: $10.24267 / 23897325.178$

- Calleja Fernández, A., Vidal Casariego, A., Cano Rodríguez, I., \& Ballesteros Pomar, M. D. (2016). Cuestionario semicuantitativo para la valoración de la ingesta dietética del paciente hospitalizado: Una herramienta sencilla para 
la práctica clínica. Nutrición Hospitalaria, 33(2), 199-511. doi: 10.20960/nh.112

- Cuervo, M., Ansorena, D., García, A., González Martínez, M. A, Astiasarán, I., \& Martínez, J. A. (2009). Valoración de la circunferencia de la pantorrilla como indicador de riesgo de desnutrición en personas mayores. Nutrición Hospitalaria, 24(1), 63-67. Recuperado de http://scielo.isciii.es/pdf/nh/ v24n1/original8.pdf

- Deossa-Restrepo, G. C., Restrepo-Betancur, L. F., VelásquezVargas, J. E., \& Varela-Álvarez, D. (2016). Evaluación nutricional de adultos mayores con el Mini Nutritional Assessment: MNA. Universidad y Salud, 18(3), 494-504. doi: 10.22267/rus.161803.54

- García Rodríguez, M., García Vilau, O., \& Odio Collazo, A. (2017). Metodología para el diagnóstico de la seguridad alimentaria y nutricional desde los gobiernos locales en un municipio. Retos de la Dirección, 11 (2), 22-37. Recuperado de http://scielo.sld.cu/pdf/rdir/v1 ln2/rdir03217.pdf

- García Salcedo, J. J., Serrano Gallardo, L. B., Recio Vega, R. B., \& Calderón Salinas, J. V. (2011). Tolerancia a la glucosa en personas mayores de 60 años. REB, 30(1), 3-11. Recuperado de https://www.medigraphic.com/pdfs/revedubio/reb$2011 /$ rebl11b.pdf

- González-Pérez, S. P., Raya-Giorguli, G., Botello-Pacheco, R., \& Rangel-Peniche, D. B. (2013). Composición corporal y dieta de mujeres adultas mayores sanas del municipio de Querétaro. Ciencia UAQ, 1-8.

- Herazo-Beltrán, Y., Quintero-Cruz, M. V., Pinillos-Patiño, Y., García-Puello, F., Núñez-Bravo, N., \& Suarez-Palacio, D. (2017). Calidad de vida, funcionalidad y condición física en adultos mayores institucionalizados y no institucionalizados. Revista Latinoamericana de Hipertensión, 12 (5), 174-181. Recuperado de https://www.redalyc.org/pdf/1702/170254309009.pdf

- Interiano Rodas, E. A., \& Valdez, R. (2017). Asociación entre desnutrición y dependencia en el adulto mayor que asiste a consulta en el Instituto Guatemalteco de Seguridad Social del departamento de Escuintla (Tesis de maestría). Recuperada de http://biblioteca.galileo.edu/tesario/ handle/123456789/796

- Landi, F., Liperoti, R., \& Onder, G. (2013). The uselfulness of anthropometric measures. European Journal of Nutrition, 52, 1683. doi: 10.1007/s00394-013-0550-6

- Méndez Estévez, E., Romero Pita, J., Fernández Domínguez, J., Troitiño Álvarez, P., García Dopazo, S., Jardón Blanco, M., ... Menéndez, M. (2013). ¿̇Tienen nuestros ancianos un adecuado estado nutricional? ¿̇ Influye su institucionalización? Nutrición Hospitalaria, 28(3), 903-913. doi: 10.3305/nh.2013.28.3.6349

- Merodio Pérez, Z., Rivas Acuña, V., \& Martínez Serrano,
A. (2015). Percepción del apoyo familiar y dificultades relacionadas con la diabetes en el adulto mayor. Horizonte Sanitario, 14(1), 14-20. Recuperado de https://www.redalyc. org/articulo.oa?id=457844964003

- Osuna-Padilla, I. A., Verdugo-Hernández, S., Leal-Escobar, G., \& Osuna-Ramírez, I. (2015). Estado nutricional en adultos mayores mexicanos: Estudio comparativo entre grupos con distinta asistencia social. Revista Española de Nutrición y Dietética, 19(1), 12-20. doi: 10.14306/renhyd.19.1.119

- Peña-Ordóñez, G. G., Bustamante-Montes, L. P., RamírezDurán, N., Halley-Castillo, E., \& García-Cáceres, L. (2016). Evaluación de la ingesta proteica y la actividad física asociadas con la sarcopenia del adulto mayor. Revista Española de Nutrición Humana y Dietética, 20(1), 16-22. doi: 10.14306/renhyd.20.1.178

- Rodríguez Quintana, T., Ayala Chauvin, M. A., Ortiz Rosero, M. F., Ordoñes Paccha, M. B., Fabelo Roche, J. R., \& Iglesias Moré, S. (2019). Caracterización de las condiciones de salud de los adultos mayores en centros geriátricos de la ciudad de Loja, Ecuador, 2017. Revista Habanera de Ciencias Médicas, 18(1), 138-149. Recuperado de http://scielo.sld.cu/scielo. php?script=sci_arttext\&pid=\$1729-519X2019000100138\&lng= es\&tlng=es.

- Zayas Somoza, E., Fundora Álvarez, V., \& Santana Porbén, S. (2018). Sobre las interrelaciones entre la sarcopenia, el envejecimiento y la nutrición. Revista Cubana de Alimentación y Nutrición, 28(1), 152-176. Recuperado de https://www.medigraphic.com/pdfs/revcubalnut/can-2018/ can1811.pdf 